\title{
Analgesia obstétrica con estimulación eléctrica transcutánea (TENS)
}

\author{
Edgar Cancino*; Pío Iván Gómez S.**
}

\section{RESUMEN}

Los métodos convencionales de analgesia obstétrica no están exentos de contraindicaciones y riesgos para la madre o el fruto de la gestación, por eso el objetivo de este trabajo es evaluar la estimulación eléctrica transcutánea (TENS) como método no invasivo de analgesia.

MATERIALES Y METODOS: Se trata de un estudio prospectivo, descriptivo que toma como Universo 18 pacientes primigestantes en fase activa de trabajo de parto a quienes se coloca TENS (Meditronic 7775) con 4 electrodos paralelos paravertebrales con frecuencia de 100 Hertz y amplitud de pulso de 100 milisegundos. Las mismas pacientes sirvieron de grupo control, al evaluar alternativamente cada hora, el grado de dolor con el TENS prendido y apagado, sin que la paciente lo supiera. Se utilizó la escala alfanumérica de 0-10 para evaluación de intensidad de dolor.

RESULTADOS: Once pacientes $(61,11 \%)$ refirieron disminución moderada, $3(16.60 \%)$ disminución leve y una (5,55\%) disminución excelente; aumento del dolor, refirieron 2 pacientes $(11.11 \%)$ y no modificación una paciente $(5.55 \%)$.

16 pacientes (88.89\%) manifestaron interés de usar TENS en una futura gestación. El apgar de los neonatos no se alteró por el uso de TENS.

No se encontró disminución del dolor en los períodos en que el TENS estuvo apagado, ni hubo alteraciones locales en los sitios de aplicación de los electrodos.

CONCLUSIONES: Consideramos que esta tecnología no invasiva, es eficaz, fácil de usar y no altera el bienestar de la madre o el fruto, por lo cual se recomienda difundir su conocimiento y evaluar series más grandes.

\section{SUMMARY}

The conventional methods of obstetric analgesia are not excent of contraindications and risks for the pregnant woman and her offspring. The objective of this study is the evaluation of the

Transcutaneous electric nerve stimulation (TENS), like non-invasive method of analgesia.

MATHERIAL AND METHODS: It's a prospective and descriptive study. 18 patients were included in an active phase of labor. All patients used a TENS (Meditronic 7775) with 4 paravertebrals paralels electrods with a 100 hertz of frecuency and 100 miliseconds of pulse amplitude. The same patients were the control group. Every hour we evaluated the grade of pain with TENS and without it. Alphanumeric scale from 0 to 10 was used for pain evaluation.

RESULTS: 11 patients $(61.11 \%)$ related moderate improvement; $3(16.60 \%)$ less reduction of pain and $1(5.55 \%)$, excellent reduction of the symptoms; a worsening pain was experimented for two patients (11.11\%); without modifications of signs and symptoms one patient $(5.55 \%)$.

16 patients ( $88.89 \%$ ) showed desire of use TENS in next pregnancy. There wasn't change on apgar's score using TENS. We didn't find improvement of the pain without TENS; and it wasn't any local alterations at the electrodes placement.

CONCLUSIONS: We believe that TENS it's a non-invasive, safety and easy technology to use that not make any disturbance on both: mother-fetus; and need more knowledge and further evaluation.

\section{Introducción}

El dolor asociado con el trabajo de parto se ha constituido en un reto para la Medicina contemporánea, por ende la ciencia ha dirigido sus investigaciones hacia el

\footnotetext{
* Gineco-Obstetra Instituto Materno Infantil de Santafé de Bogotá D.C.

Profesor Asociado. Universidad Nacional de Colombia. Coordinador Clínica de Dolor. Instituto Materno Infantil de Santafé de Bogotá, D.C.
}

descubrimiento de sustancias y técnicas que proporcionen alivio al dolor de la mujer gestante en trabajo de parto sin que se afecte el binomio Madre-hijo ni se trastorne el trabajo de parto.

Se han utilizado diferentes métodos convencionales para disminuir el dolor durante el trabajo de parto y parto incluyendo analgésicos y sedantes, analgesia peridural, bloqueos anestésicos locales, acupuntura, métodos psicoprofilácticos y estimulación eléctrica transcutánea, pudiendo algunos de estos afectar el binomio: Madre-Feto. 
El proceso de selección del mejor método analgésico durante el trabajo de parto no es fácil y se requiere de una evaluación completa, que incluya el examen del estado físico, la anamnesis, el deseo de la paciente y valorar los riesgos frente a los beneficios. La analgesia y la anestesia ideales serían aquellas que proporcionen un alivio excelente del dolor, que no modifiquen el curso normal del trabajo de parto, que no ejerzan efectos indeseables en el feto ni en la madre y que sean de fácil administración.

La estimulación eléctrica transcutánea es un método no invasivo para manejo del dolor, el cual está basado principalmente en la teoría de la compuerta de control del dolor propuesta por los Drs. Melzack y Wall o en la estimulación de la liberación local de endorfinas (1-2).

La estimulación eléctrica transcutánea ha sido utilizada durante cerca de 18 años en el manejo de una gran variedad de síndromes dolorosos, agudos y crónicos en diferentes campos de la Medicina. Este método ha mostrado ser seguro y efectivo durante el trabajo de parto y el parto, sin efectos sobre la madre o el feto (3-8).

\section{Vías de dolor en el trabajo de parto}

El dolor del parto se origina en los nociceptores de las estructuras uterinas y perineales. Durante el primer estadio del trabajo de parto las fibras nerviosas que transmiten la sensación dolorosa de las contracciones uterinas y de la dilatación del cervix son las que transcurren con las fibras simpáticas y entran en el neuroeje a nivel de los segmentos medulares $\mathrm{T}_{10}, \mathrm{~T}_{11}, \mathrm{~T}_{12}$ y $\mathrm{L}_{1}$.

El dolor causado por la dilatación del canal del parto, de la vulva y el periné, es transmitido a través de los nervios pudendos los cuales entran al neuroeje a través de las raíces dorsales de los segmentos: $\mathrm{S}_{2}, \mathrm{~S}_{3}$ y $\mathrm{S}_{4}$.

Las fibras nerviosas que transmiten dolor suprapúbico no han sido aún identificadas, a este nivel hay una inervación sensitiva superpuesta que está a cargo del nervio abdominogenital menor del plejo lumbar que llega al periné por el conducto inguinal.

\section{Estimulación nerviosa eléctrica transcutánea}

La estimulación nerviosa eléctrica transcutánea es una forma no invasiva de manejo del dolor; a pesar de que el mecanismo exacto de acción para aliviar el dolor de este método no es claro, la mas probable forma de acción es la que involucra la teoría de Melzack y Wall basada en el mecanismo de la compuerta medular (1). Esta noción fue tomada de datos electrofisiológicos de experimentos en animales que han demostrado efectos diferentes de los axones colaterales de las fibras aferentes de grandes diámetros que llevan información de tacto y presión y de las fibras aferentes de diámetro pequeño las cuales llevan impulsos nocioceptivos hasta las interneuronas dentro de la sustancia gelatinosa. Estas interneuronas pueden ser facilitadas a través de las fibras colaterales aferentes de gran diámetro e inhibidas a través de los axones colaterales del sistema de fibras de pequeño diámetro. Además las interneuronas son inhibitorias en las terminales de ambos tipos de fibras aferentes. Esta teoría de la compuerta estuvo sujeta a múltiples críticas ya que conceptualmente no podía explicar la disminución del dolor en una gran variedad de condicio- nes clínicas, sin embargo sigue siendo la más aceptada y se ha convertido en la base para muchas explicaciones contemporáneas de disminución del dolor a través de la estimulación eléctrica transcutánea.

Indudablemente se requieren más estudios que involucren técnicas histoquímicas y electrofisiológicas para explicar y comprender mejor cómo la estimulación eléctrica transcutánea modula la percepción del dolor.

La estimulación eléctrica transcutánea ha sido utilizada en los últimos 20 años en el manejo de una gran variedad de síndromes dolorosos agudos y crónicos como método no invasivo de manejo del dolor (10-12, 16-21).

\section{TENS y trabajo de parto}

La estimulación eléctrica transcutánea ha sido usada especialmente en Europa y en menor porcentaje en USA, para controlar el dolor agudo asociado con el trabajo de parto y el parto. Jones (13) reportó que $82 \%$ de las pacientes en trabajo de parto que usaron el TENS presentaron disminución substancial del dolor lumbar y $75 \%$ presentaron disminución significativa del dolor abdominal del trabajo de parto durante la primera fase del trabajo de parto.

Bundsen y cols (7) evaluando el efecto analgésico del TENS durante el parto y sus efectos en las condiciones neurológicas y bioquímicas del neonato, encontraron disminución de intensidad del dolor lumbar durante el parto sin producir efectos adversos en el neonato.

Robson (14) notó que la estimulación suprapúbica produjo significativa disminución del dolor ventral.

Grim y Morey (15) seleccionaron 15 pacientes para evaluar la efectividad del TENS en el manejo del dolor del trabajo de parto, encontrando que $87 \%$ de las participantes referían algún grado de disminución del dolor y la quinta parte refirieron excelente disminución del dolor, además la mayoría de participantes expresaron el deseo de usar el TENS en su siguiente parto; los autores recomiendan usar frecuencias de $100 \mathrm{~Hz}$ y amplitud de pulso de 100 Milisegundos, el cual es mas asociado con estimulación de fibras A. En el estudio de Harrison y colaboradores (4) no se encontraron efectos colaterales a la terapia y además se reportó que el TENS no interfería en la monitoría electrónica fetal.

\section{Objetivos}

- Evaluar la efectividad de la estimulación eléctrica transcutánea como método alternativo no invasivo del manejo del dolor durante el trabajo de parto y las posibles repercusiones con el uso del TENS en mujeres colombianas en fase activa del trabajo de parto y en el Binomio: Madre-Hijo.

Determinar el deseo de la paciente de usar este método de analgesia en posteriores gestaciones.

\section{Materiales y métodos}

Se trata de un estudio descriptivo prospectivo en el que se incluyeron 18 pacientes primigestantes que ingresaron en fase activa del trabajo de parto al Instituto Materno Infantil de Santafé de Bogotá entre el 15 de diciembre de 1993 y 30 de enero de 1994 y no recibieron ningún tipo de analgesia a excepción del TENS. Se les 
instruyó sobre el tipo de estudio y la utilidad del TENS y se les solicitó su consentimiento.

Se tomó como fuente de información la Historia clínica materna y del fruto, recolectando los datos en un formulario precodificado.

El dolor se evaluó cada hora en el trabajo de parto según la escala alfanumérica subjetiva de 0 a 10 , siendo 0 la ausencia de dolor y 10 el dolor mas intenso, correlacionándose el grado de disminución con los términos de leve, moderada o severa.

Se utilizó un estimulador eléctrico transcutáneo marca "Meditronic", modelo 7725. Se utilizaron electrodos de carbono ubicados en forma paralela y paravertebral a nivel de los dermatomas $T_{10}$ a $L_{1}$ y $S_{2}$ a $S_{4}$ a $5 \mathrm{~cm}$ de la espina dorsal. Una vez ubicados los electrodos, la unidad se fijó en una frecuencia de $100 \mathrm{~Hz}$ y una amplitud de pulso de 100 milisegundos y la intensidad varió de 20 a 30 miliamperios de acuerdo con la tolerancia de cada paciente. En cada canal se incrementó su amplitud desde 0 hasta el nivel mas bajo percibido por la paciente y se mantuvo así en cada canal hasta que la contracción uterina apareció y en este momento se aumentó la amplitud de la unidad tan alto como lo toleró confortablemente la paciente durante toda la contracción.

Los niveles de estimulación bajos y altos se aumentaron a medida que transcurrió el trabajo de parto.

Se realizó control y evaluación habitual del trabajo de parto, según las normas de la Institución.

Para evaluar la efectividad del TENS se apagó durante la segunda, cuarta, sexta, octava... horas de uso en el trabajo de parto, sin hacérselo saber a la paciente y se dejó prendido en la primera, tercera, quinta, séptima, novena... horas.

Se reinterrogó a las pacientes en el postparto inmediato, a las 12 y 24 horas en el puerperio.

\section{Resultados}

Se aplicó TENS a 18 pacientes, las cuales tuvieron todas parto vaginal, una paciente requirió espátulas de rotación y desprendimiento por una variedad de posición posterior.

La duración del trabajo de parto estuvo dentro de los límites normales de acuerdo al partograma de la Institución.

Un feto presentó bradicardia en el expulsivo, el cual era fruto de una paciente con hipertensión inducida por la gestación, encontrándose circular apretada al cuello y un desprendimiento placentario del $5 \%$.

El Apgar dado por el pediatra al minuto del nacimiento fue de $8 / 10$ en 15 neonatos $(83.33 \%), 7 / 10$ en dos $(11.11 \%)$ y $6 / 10$ en uno $(5.55 \%)$.

De las 18 pacientes, una refirió disminución excelente del dolor $(5.55 \%)$, once pacientes disminución moderada $(61.11 \%)$ y tres una disminución leve $(16.6 \%)$.
Dos $(11.11 \%)$ reportaron incremento del dolor y una (5.5\%) no modificación del mismo. Diez y seis pacientes $(88.89 \%)$ manifestaron su deseo de utilizar el TENS en una futura gestación, siendo una de ellas la que no reportó analgesia con su uso, pero refería el interés de usarlo porque "disminuyó el tiempo de trabajo de parto".

No se encontró modificación del puntaje subjetivo en ninguna paciente cuando el TENS se dejó apagado durante las contracciones.

No se encontró ningún efecto colateral en el sitio de aplicación de los electrodos.

\section{Discusión}

El dolor es un fenómeno complejo asociado a factores de toda índole, desde diferencias socioculturales en la percepción del dolor hasta variaciones individuales en la percepción y repercusiones del mismo.

Intentar separar los mecanismos neurofisiológicos de los psicológicos que intervienen en la disminución del dolor no es fácil y desde el punto de vista práctico carece de importancia, por tal razón este estudio se realizó autocontrolado por el paciente, el cual evaluaba la efectividad del TENS. La respuesta es subjetiva y puede verse influenciada por muchos factores, entre ellos el efecto placebo de la simple colocación del aparato; de todas formas y aunque la muestra presente es pequeña, vemos como de 18 pacientes, quince $(83.33 \%$ ), reportaron disminución del dolor en el trabajo de parto, hallazgo similar al encontrado en otros estudios donde los porcentajes varían de $82 \%$ a $85 \% .(4,7,13-15)$.

La duración del trabajo de parto en todas las pacientes estuvo dentro de la normalidad siguiendo el partograma de la institución.

No se encontró ninguna repercusión sobre el bienestar fetal, asociada al uso de TENS, teniendo todos los neonatos Apgar por encima o igual a $6 / 10$, siendo el $94.45 \%$ mayor de $7 / 10$.

\section{Conclusiones}

El resultado de este trabajo concuerda con las demás publicaciones al respecto, mostrando grandes ventajas de la estimulación eléctrica transcutánea como un método efectivo para tener en cuenta en el arsenal analgésico que debemos tener disponible dentro de la práctica obstétrica; es un método efectivo, no invasivo, no costoso, seguro tanto para la madre como para el fruto de la gestación, de fácil administración y control.

Sugerimos incrementar el uso de estimuladores eléctricos transcutáneos en las salas de maternidad del país para aumentar nuestro conocimiento de esta tecnología y conocer otras experiencias al respecto.

\section{BIBLIOGRAFIA}

1. Melzack R. Wall P. Pain Mechanisms. A new theory. Science 1965; 150: $971-979$.

2. Sjolund B., Terenius L., Ericksonn M. Increased cerebrospinal fluid levels of endorphines after electroacupunture. Acta Physiol Scand 1977; 100: 382- 384 .
3. Roth GM., Wolf SL. Applications of transcutaneous electrical nervy stimulation in the management of patients with pain. Phys Ther 1985; 65: $314-322$

4. Harrisow R. et al. Pain Relief in labor using transcutaneous electrical nerve stimulation (TENS)/. A TENS/TENS placebo controlled study in two parity groups. Br J Obstet Gynaecol 1986; 93: 739-746. 
5. Shealy CW., Maurer D. Transcutaneous nerve stimulation for control of pain. Surg Neurology 1974; 2: 45-47.

6. Bundsen P., Erickson K., Peterson L. et al. Pain relief in labor by transcutaneous electrical nerve stimulation. Acta Obstet Gynecol Scand 1982; 61: 129-136.

7. Bundsen P., Erickson K., Peterson L. E. et al. Pain relief in labor by transcutaneous electrical nerve stimulation. Testing of a modified stimulation technique and evaluation of the neurologycal and biochemical condition of the newborn infant. Acta Obstet Gynaecol Scand 1982; 61: 289-297.

8. Robson JE. Transcutaneous nerve stimulation for pain relief in labor. Anaesthesia 1979; 34: 357-360.

9. Bosbaum A., Fields HL. Endogenous pain control mechanisms: Review and hypotesis. Ann Neurol 1978; 4: 451-462.

10. Griego, Montoya. Analgesia y anestesia obstétrica. Pontificia Universidad Javeriana. Hospital Universitario San Ignacio. 1992.

11. Solomon RA., Viernstein MC., Long D.M.: Reduction of postoperative pain and arcotic use by transcutaneous electrical nerve stimulation. Surgery 1990; 87: 142-146.

12. Schomburg FL., Carter et al. Transcutaneous electrical nerve stimulation for postlaparotomy pain. Phys Ther 1983; 63: 188-193.
13. Jones MC. Transcutaneous nerve stimulation in labor. Anaesthesia 1980; 35: 372-375.

14. Robson JE. Forum: Transcutaneous nerve stimulation for pain relief in labour. Anaesthesia 1979; 34: 357-360.

15. Grim L, Morey S. Transcutaneous electrical nerve stimulation for relief of parturition pain. A clinical report. Phys Ther 1985; 65: 337-340.

16. Wolf SL., Gersh MR., Rao VR. Examination of electrode placements and stimulating parameters in treating chronic pain with conventional transcutaneous nerve stimulation. Pain 1981; 11: 37-47.

17. Cthia YA. et al. Effectiveness of transcutaneous electric nerve stimulator for pain relief in labor. Asia-Oceanía J. Obstet. Gynecol. 1990; 16(2):145-151

18. Johansson F. et al. Predictors for the outcome of treatment with high frecuency transcutaneous electrical nerve stimulation in patients with chronic pain. Pain 1980; 9: 55-61.

19. Crawford, Selwyn J. General analgesia and anaesthesia in Obstetrics. Clinics in anaesthesiology 1986; 4(1).

20. Ali J. et al. The effect of transcutaneous electrical nerve stimulation on postoperative pain and pulmonary function. Surgery 1981; 89: 507-512.

21. Riley J.E. The impact of TENS on the postcesarean patient. J Obstet. Gynecol. and Neonatal Nursing 1982; 11: 325-329.
Correos de Colombia

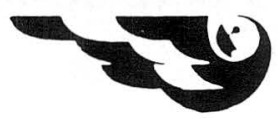

- SERVICIO ENCOMIENDAS ASEGURADAS

- SERVICIO CARTAS ASEGURADAS

- SERVICIO DE GIROS

- BUROFAX

- SERVICIO "CORRA"

- SERVICIO DE CORREO ORDINARIO

\section{Estos son nuestros servicios jutilícelos!}

\author{
- SERVICIO DE CORREO CERTIFICADO \\ - SERVICIO DE CERTIFICADO ESPECIAL \\ - ENCOMIENDAS CONTRA REEMBOLSO \\ - SERVICIO TARIFA POSTAL REDUCIDA \\ - SERVICIO DE FILATELIA \\ - SERVICIO ELECTRONICO \\ - SERVICIO INTERNACIONAL APR/SAL \\ - SERVICIO RESPUESTA COMERCIAL \\ - SERVICIOS ESPECIALES
}

Teléfonos para quejas y reclamos: 3340304 - 3415536 - Santafé de Bogotá, D.C. - Colombia Cuente con nosotros hay que creer en los Correos de Colombia 